\title{
OPERACIÓN DE UNA ESTACIÓN DE BOMBEO CON GRUPOS ELECTRÓGENOS
}

\author{
Ruiz Padín, A. ${ }^{1}$, Medina Martínez, J.M. ${ }^{2}$ \\ ${ }^{1}$ Departamento de Ingeniería y Ciencias Agrarias, Universidad de León, aruip@unileon.es \\ 2 Director Técnico, Comunidad de Regantes del Canal del Pisuerga. Astudillo, Palencia.
}

\section{Resumen}

Durante los meses de mayo y junio de 2011, la energía eléctrica para el bombeo del sector D (644 ha regadas) de la Zona Regable del Canal del Pisuerga (en adelante, ZR), en Palencia y Burgos, fue suministrada con grupos electrógenos (en adelante GE) debido al robo de los transformadores de la estación. Durante el período de operación con GE se tomaron lecturas de energía consumida por las bombas, energía consumida por los circuitos auxiliares, agua bombeada, horas de funcionamiento y volúmenes de repostaje de cada uno de los GE, y superficies, volúmenes suministrados y cultivos para los hidrantes operados durante el período. Con estos datos, se determinaron los consumos totales, consumos específicos y rendimientos de los grupos; consumos por $\mathrm{m}^{3}$ bombeado y por ha regada; costes fijos y variables de operación con GE; y costes fijos y variables de operación con energía de la red. El principal problema durante la operación fue la limitada autonomía de los equipos. El coste final del agua de riego, para el período analizado, fue de $0,165 € \mathrm{~m}^{-3}$, frente a los $0,026 € \mathrm{~m}^{-3}$ con suministro de la red eléctrica para esa campaña.

\section{Abstract}

During the months of May and June of 2011, electricity for pumping sector D (644 irrigated ha) of the Pisuerga Channel Irrigation Area (hereinafter ZR), in Palencia and Burgos, Spain, was supplied with generators (hereinafter GE) due to theft of the pumping station transformers. During the operation with GE, readings of energy consumed by pumps, energy consumed by the auxiliary circuits, pumped water, hours of operation and refueling volumes for each GE, and surfaces, volumes supplied and crops for hydrants operated, were taken during the period.

With these data, total consumption, specific consumption and GE performance were determined; consumption per pumped cubic meter and per watered hectare; fixed and variable costs of operation with GE; and fixed and variable costs of operation with utility power. The main problem during the operation was limited autonomy of GE. The final cost of irrigation water for the analyzed period was $0.165 € \mathrm{~m}^{-3}$, compared with $0.026 € \mathrm{~m}^{-3}$ with utility power for that campaign.

\section{1- Introducción y objetivos}

La ZR comprende la superficie dominada por el canal del Pisuerga en tierras de los términos de Astudillo, Villalaco, Villodre, Melgar de Yuso, Itero de la Vega, Lantadilla, Zarzosa, Ventosa y Herrera del Pisuerga, en la provincia de Palencia; y San Llorente de la Vega y Melgar de Fernamental, en la provincia de Burgos. El canal domina una superficie de 12.000 ha, de las cuales se riegan 9.530. La zona regable está en proceso de modernización, habiéndose concluido la mejora de 6.493 ha, que se encuentran en explotación desde el año 2009. 
En abril de 2011, iniciada la campaña de riegos, se produjo el robo de los devanados de cobre de los transformadores de la estación de bombeo correspondiente al sector $D$ de la $Z R$, dejando esta inútil para el servicio. El plazo de entrega de los nuevos transformadores se demoraba hasta principios de julio, por lo que hubo de alistarse la estación para su funcionamiento mediante GE para no interrumpir la campaña.

El objetivo del presente trabajo es describir la solución adoptada y su problemática asociada, cuantificar los factores implicados en el coste del riego con energía de GE en una estación modernizada, fijos y variables, y determinar el sobrecoste sobre la energía de red.

\section{2- Materiales y métodos}

El sector $D$ tiene una superficie de 1.183 ha, de las que en la campaña 2011 se regaron 644 , cultivadas principalmente de cereal de invierno y remolacha (Tabla 1).

Tabla 1. Distribución de cultivos

\begin{tabular}{rrrrrrrr}
\hline C. inv. & Rem. & Alfalfa & Girasol & C. biom. & Maíz & Otras & Total \\
\hline 328,5 & 118,0 & 68,0 & 63,8 & 25,8 & 23,1 & 16,2 & $\mathbf{6 4 3 , 5}$ \\
\hline $51,05 \%$ & $18,34 \%$ & $10,57 \%$ & $9,92 \%$ & $4,01 \%$ & $3,59 \%$ & $2,52 \%$ & $\mathbf{1 0 0 , 0 0 \%}$ \\
\hline
\end{tabular}

C. inv.: Cereales de invierno; Rem.: Remolacha; C. biom.: Cultivo para biomasa. Superficies en ha.

La campaña de riegos comenzó el 1/04/11. El período de riego con GE se extendió desde el 12/05/11 hasta el 09/07/11, con una duración de 58 días, prosiguiendo la campaña hasta el 30/09/11 con energía de la red tras la instalación de los nuevos transformadores.

La estación de bombeo del sector $\mathrm{D}$ consta de 4 bombas verticales principales de 350 $\mathrm{kW}$ y 2 bombas verticales auxiliares de $200 \mathrm{~kW}$, operables mediante variador de frecuencia. Las instalaciones necesarias para adaptar el funcionamiento de la estación al suministro mediante GE consistieron en:

- Suministro y puesta en marcha de dos GE de 800 kVA cada uno para suministro de energía a las bombas, en régimen de alquiler.

- Suministro y puesta en marcha de un GE de 30 kVA para suministro de energía a los circuitos auxiliares, en régimen de alquiler.

- Suministro y puesta en marcha de un transformador de 400 kVA, relación 400:690, en régimen de alquiler.

- Tendido provisional de una línea desde los grupos principales hasta el embarrado general de potencia de las bombas.

- Tendido provisional de una línea desde el grupo auxiliar hasta el embarrado de circuitos auxiliares.

- Conexionado de los equipos y cuadros de protección respectivos.

El alquiler de los equipos se facturó por días, del 4/05/11 al 9/07/11, totalizando 65 días. Dado que en 2011 solo se regaba aproximadamente la mitad del sector, y de ésta más de la mitad correspondía a cereales de invierno, durante la mayor parte del tiempo solo se 
operó una de las bombas principales. La potencia de grupos instalada fue claramente excesiva (del orden del cuádruple de la requerida). Con un solo grupo de $400 \mathrm{kVA}$ se hubiera satisfecho la demanda punta con un resguardo aceptable. Cabe reseñar que la decisión de alquilar dos grupos se tomó para garantizar la disponibilidad de reserva en caso de fallo, y su tamaño por razones de autonomía, no por potencia.

Con el fin de permitir un análisis detallado de la operación de la estación en el período, se recogieron con periodicidad de 12 horas lecturas de los siguientes parámetros:

- Lecturas de energía consumida por las bombas, mediante analizador de redes aguas arriba del embarrado de potencia.

- Lecturas de energía consumida por los circuitos auxiliares (iluminación de la estación, válvulas motorizadas, alimentación de autómatas, alarmas, etc.), mediante analizador de redes a la salida del grupo auxiliar.

- Lecturas de agua bombeada, mediante el caudalímetro electromagnético del colector de impulsión.

- Horas de funcionamiento de cada uno de los grupos, en cada uno de sus cuadros de mando.

- Volumen de repostaje de cada uno de los grupos (en el momento del repostaje).

- Superficies, volúmenes suministrados y cultivos para los hidrantes operados durante el período.

Se recogieron también todos los datos contables necesarios para el análisis de costes, principalmente alquileres de equipos, portes, tendido de lineas provisionales, importe de los repostajes y facturas eléctricas durante el total de la campaña.

Con los datos citados, se determinaron los consumos totales, consumos específicos y rendimientos de los grupos; consumos por $\mathrm{m}^{3}$ bombeado y por ha regada; costes fijos y variables de operación con GE; y costes fijos y variables de operación con energía de la red.

\section{3- Resultados y discusión}

Los totales de las lecturas realizadas figuran en el cuadro que sigue. 


\section{Lecturas analizadores de red}

\begin{tabular}{lrl}
\hline $\begin{array}{l}\text { Servicios auxiliares } \\
\text { Potencia }\end{array}$ & $9.178,07$ & $\mathrm{~kW} \cdot \mathrm{h}$ \\
& $235.315,56$ & $\mathrm{kWh}$ \\
Lectura caudalimetro & $771.555,00$ & $\mathrm{~m}^{3}$ \\
\hline & & \\
Lecturas horas de funcionamiento & 349,00 & $\mathrm{~h}$ \\
\hline Grupo izquierdo & 425,00 & $\mathrm{~h}$ \\
Grupo derecho & $1.392,00$ & $\mathrm{~h}$ \\
Grupo auxiliar & & \\
& & \\
Lecturas repostaje & $39.494,00$ & $\mathrm{I}$ \\
\hline Grupo izquierdo & $41.052,00$ & $\mathrm{I}$ \\
Grupo derecho & $4.397,00$ & $\mathrm{I}$
\end{tabular}

Durante el período de operación con GE el caudal continuo medio efectivo bombeado fue de $0,277 \mathrm{~m}^{3} \mathrm{~s}^{-1}$, que supone un caudal medio de $0,154 \mathrm{~m}^{3} \mathrm{~s}^{-1}$ durante la operación con GE para el índice de funcionamiento del $55,6 \%$ de la estación. La dotación media correspondiente fue de $0,24 \mathrm{I} \mathrm{s}^{-1} \mathrm{ha}^{-1}$, que resulta baja debido a la elevada superficie de cereales de invierno.

Los parámetros de funcionamiento calculados figuran en el cuadro que sigue.

\begin{tabular}{lrl} 
Parámetros grupos potencia & & \\
\hline Consumo horario grupo izquierdo & $113,16 \mathrm{I} \mathrm{h}^{-1}$ \\
Consumo horario grupo derecho & $96,59 \mathrm{I} \mathrm{h}^{-1}$ \\
Consumo total de combustible & $80.546 \quad \mathrm{l}$ \\
Consumo de gasoil por UEE* & $0,342 \mathrm{I} \mathrm{kW}^{-1} \mathrm{~h}^{-1}$ \\
Consumo específico* & $284,8 \mathrm{~g} \mathrm{~kW}^{*} \mathrm{~h}^{-1}$ \\
Consumo de energía por UVB & $0,305 \mathrm{~kW} \mathrm{~h} \mathrm{~m}^{-3}$ \\
Consumo de gasoil por UVB* & $0,104 \quad \mathrm{I} \mathrm{m}^{-3}$ \\
\hline Potencia activa media demandada* & $304,03 \mathrm{~kW}$ \\
Rendimiento total motor-generador & $29,3 \%$ \\
\hline
\end{tabular}

Parámetros grupo auxiliar

\begin{tabular}{lrl} 
Consumo horario & 3,16 & $\mathrm{I} \mathrm{h}^{-1}$ \\
Consumo de gasoil por UEE & $0,479 \mathrm{I} \mathrm{kW}^{-1} \mathrm{~h}^{-1}$ \\
Consumo específico & $398,6 \mathrm{~g} \mathrm{~kW}^{-1} \mathrm{~h}^{-1}$ \\
Repercusión de gasoil por UVB & $0,006 \quad \mathrm{I} \mathrm{m}^{-3}$ \\
\hline Potencia activa media demandada & $19,63 \quad \mathrm{~kW}$ \\
Rendimiento total motor-generador & $20,96 \%$ \\
\hline
\end{tabular}

* Valor medio para los dos grupos

UEE: unidad de energía eléctrica, UVB: unidad de volumen bombeada

Para el cálculo del consumo específico se ha tomado para el gasoil una densidad a 20 ${ }^{\circ} \mathrm{C}$ de $832 \mathrm{~kg} \mathrm{~m}^{-3}$ y un poder calorífico inferior de $43.100 \mathrm{~kJ} \mathrm{~kg}^{-1}$. 
Los grupos de potencia funcionaron de forma alterna, nunca simultáneamente, permitiendo alargar el intervalo entre repostajes. De todas formas, debido a la imposibilidad de disponer un depósito provisional de alta capacidad por razones administrativas hizo que fuera necesario repostar desde camión cisterna diariamente, alternando los grupos de madrugada. La potencia media demandada indica que el factor de carga medio del grupo en funcionamiento fue del $41,3 \%$ (para un cos $\phi$ de 0,92 ), lo que confirma el exceso de capacidad de generación anteriormente reseñado.

El consumo específico de los grupos de potencia es aceptable, aunque superior al de grupos de alta eficiencia (alrededor de $230 \mathrm{~g} \mathrm{~kW}^{-1} \mathrm{~h}^{-1}$ ). Debe tenerse en cuenta que los grupos funcionaron la mayor parte del tiempo por debajo de media carga, lejos de la zona de mayor eficiencia. El consumo específico del grupo auxiliar es elevado, con un rendimiento casi diez puntos inferior al de los grupos de potencia. Este bajo rendimiento es explicable por su funcionamiento continuo, mucho tiempo a carga casi nula.

El consumo total de gasoil por UVB, incluyendo la repercusión del funcionamiento de grupos auxiliares, fue de $0,110 \mathrm{I} \mathrm{m}^{-3}$.

En el cuadro que sigue figura el detalle desglosado de los costes fijos y variables de operación con GE, así como los costes unitarios. Todos los costes corresponden a precios de facturación sin IVA.

A.1. Alquileres y portes (65 días)

2 grupos principales de $800 \mathrm{kVA}$, a $269,27 € \mathrm{~d}^{-1} \mathrm{c} / \mathrm{u}$

1 grupo auxiliar de $30 \mathrm{kVA}$, a $9,95 € \mathrm{~d}^{-1}$

1 transformador de $400 \mathrm{kVA}, \mathrm{R} 400: 690$, a $40,25 € \mathrm{~d}^{-1}$

Portes de entrega y recogida
$40.222,52 € \quad 64,05 \% \mathrm{~s} / \mathrm{A}$

$35.004,67 € 87,03 \%$ s/A. 1

$646,60 € \quad 1,61 \%$ s/A.1

$2.616,25 € \quad 6,50 \%$ s/A.1

$1.955,00 € \quad 4,86 \%$ s/A. 1

A.2. Adaptación para funcionamiento con GE

22.578,00€

$35,95 \% \mathrm{~s} / \mathrm{A}$

Líneas provisionales, aparellaje, protección

$22.578,00 €$

B. Coste variable

$\mathbf{6 4 . 5 8 4 , 7 6 €}$

$50,70 \% \mathrm{~s} / \mathrm{C}$

Gasoil $\left(84.943\right.$ I a $\left.0,\left.76 €\right|^{-1}\right)$

$64.584,76 €$

C. Coste total

$127.385,28 €$

D. Volumen total bombeado $\left(\mathrm{m}^{3}\right)$

771.555

E. Coste unitario total $\left(€ \mathrm{~m}^{-3}\right)$

0,165

Coste unitario fijo $\left(€ \mathrm{~m}^{-3}\right)$

0,081

Coste unitario variable $\left(€ \mathrm{~m}^{-3}\right)$

0,084

$49,30 \%$ s/E

$50,70 \%$ s/E 
Sobre los costes indicados caben las las siguientes observaciones:

- El coste final por $\mathrm{m}^{3}$ bombeado depende de la altura de bombeo, por lo que debe ser empleado con esta reserva.

- La mitad de este coste se debe a costes fijos. De haber ajustado la potencia instalada a $400 \mathrm{kVA}$, como se propone más arriba, el coste unitario fijo se habría reducido a $0,047 € \mathrm{~m}^{-}$ ${ }^{3}$, y el coste unitario total a $0,131 € \mathrm{~m}^{-3}$. Es por tanto importante estimar con la mayor exactitud posible las necesidades de potencia.

- El coste fijo correspondiente a la adaptación de la instalación es irrecuperable, aunque el material se ha almacenado en previsión de posibles futuras incidencias similares y podría ser empleado en cualquiera de las estaciones de la ZR.

En comparación a los valores indicados, el coste variable del bombeo con energía de la red, calculado a partir de las facturas de suministro eléctrico del resto de la campaña fue de $0,0265 € \mathrm{~m}^{-3}$ en período P5 y $0,0196 € \mathrm{~m}^{-3}$ en período P6. Esto supone que el coste variable de bombeo con GE resulta entre 3,2 y 4,3 veces superior al coste con energía de la red. Incluyendo los costes fijos, el coste total del bombeo con energía de la red fue de $0,0307 € \mathrm{~m}^{-}$ ${ }^{3}$ en período P5 y $0,0238 € \mathrm{~m}^{-3}$ en período P6, siendo el coste total con GE entre 5,3 y 7,2 superior al coste con energía de la red.

Un análisis de las cifras anteriores permite afirmar que para una campaña completa, el coste variable del agua en caso de operar el bombeo con GE, aunque alto (en torno a los $380 €$ ha $^{-1}$ ), parece perfectamente asumible. No ocurre lo mismo con el coste total, que supondría en torno a los $740 €$ ha $^{-1}$. Dado que la probabilidad de que incidentes similares al acaecido en 2011 en la ZR parece estar aumentando, tal vez resultaría conveniente disponer de GE compartidos (p.ej. cada 100.000 ha), en régimen de propiedad o de reserva preferente con alquiler cubierto mediante seguro, u otra fórmula similar, y material de adaptación reutilizable, lo que permitiría que el coste fijo fuera casi nulo.

\section{4- Conclusiones}

Las principales conclusiones obtenidas del presente trabajo son:

- Es posible la operación de una estación de bombeo modernizada mediante grupos electrógenos debido a circunstancias sobrevenidas, y existe disponibilidad de potencia en el mercado de alquiler para ello.

- El plazo necesario para alistar la estación para comenzar el riego con energía de los grupos puede estimarse entre 5 y 7 días.

- La operación durante el período analizado se produjo sin incidencias debidas al tipo de suministro eléctrico.

- El principal problema durante la operación fue la limitación de autonomía de los equipos (apróx. 15 horas), debiéndose rotar los grupos a horas intempestivas.

- El coste final del agua de riego, para el período analizado, fue de $0,165 € / \mathrm{m}^{3}$, frente a los $0,026 € / \mathrm{m}^{3}$ con suministro de la red eléctrica para esa campaña. 